Annuaire suisse de politique de développement

$21 \mid 2002$

Agriculture suisse et mondialisation

\title{
5. Appendice
}

\section{Gérard Perroulaz, Antonio Girardi et Nicolas Roguet}

\section{OpenEdition \\ Journals}

Édition électronique

URL : http://journals.openedition.org/aspd/958

DOI : $10.4000 /$ aspd.958

ISSN : 1663-9669

\section{Éditeur}

Institut de hautes études internationales et du développement

\section{Édition imprimée}

Date de publication : 1 avril 2002

Pagination : 365-369

ISSN : 1660-5934

\section{Référence électronique}

Gérard Perroulaz, Antonio Girardi et Nicolas Roguet, «5. Appendice », Annuaire suisse de politique de développement [En ligne], 21 | 2002, mis en ligne le 07 septembre 2012, consulté le 08 septembre 2020. URL : http://journals.openedition.org/aspd/958 ; DOI : https://doi.org/10.4000/aspd.958 


\section{APPENDICE}

\subsection{SIGNES UTILISÉS, TAUX DE CHANGE ET REMARQUES}

\section{$\square$ Signes \\ - $\quad$ Nul (aucun montant) \\ $\mathrm{p}$ Provisoire}

Taux de change:

Francs suisses pour 1 dollar des Etats-Unis: n.a. Non applicable

n.d. Non disponible

$1997: 1,4500$

$1998: 1,4497$

$1999: 1,5027$

$2000: 1,6869$

\section{$\square$ Remarques:}

- Les chiffres étant arrondis, les totaux ne correspondent pas toujours à la somme des composants.

- Les marges d'erreur sont généralement bien supérieures au degré d'arrondissement des chiffres. Ceux-ci reposent souvent sur des informations partielles.

- Les principaux sigles utilisés dans cette partie statistique se trouvent dans la liste des abréviations, à la fin de l'Annuaire.

\subsection{COMPOSITION DES GROUPES DE PAYS}

Il n'existe pas de classification unique pour les statistiques des flux commerciaux, financiers et d'aide au développement. Chaque organisation internationale tend à définir ses propres catégories. Pour le commerce, nous nous sommes fondés sur la classification établie par la CNUCED sur la base du système défini par le Bureau de statistique des Nations Unies. Concernant les flux financiers et l'aide publique au développement, nous avons retenu la classification du Comité d'aide au développement (CAD) de l'OCDE' ${ }^{1}$. Le haut degré d'agrégation des données disponibles concernant les mouvements de capitaux privés ne permet pas de les classer différemment. Par conséquent, les statistiques de l'Annuaire comportent deux classifications distinctes, l'une pour la première section (commerce), l'autre pour les trois autres sections (flux financiers, aide publique au développement et relations de la Suisse avec les pays en développement et les pays en transition) $)^{2}$.

1. Le CAD est un comité spécialisé de l'OCDE. Il cherche à accroître le volume des ressources destinées aux pays en développement et à en améliorer l'efficacité.

2. Les statistiques du commerce international publiées par l'OCDE sont elles-mêmes classées selon des catégories différentes de celles du CAD. 


\section{A. STATISTIQUE DU COMMERCE INTERNATIONAL (LISTE ÉTABLIE PAR LA CNUCED)}

a) Classification générale

Pays développés à économie de marché

Europe: comprend les pays de l'Union européenne, de l'AELE, Gibraltar et les îles Féroé.

Outre-mer: Canada, Etats-Unis d'Amérique, Israël, Japon, Australie, NouvelleZélande, Afrique du Sud.

Pays d'Europe orientale

Albanie, Bélarus, Bulgarie, Estonie, Fédération de Russie, Hongrie, Lettonie, Lituanie, Pologne, République de Moldava, République tchèque, Roumanie, Slovaquie et Ukraine.

Pays du Tiers Monde

Comprend tous les autres pays et territoires du monde.

\section{b) Classification géographique des pays du Tiers Monde}

Afrique: tous les pays sauf l'Afrique du Sud.

Amérique: tous les pays sauf le Canada et les Etats-Unis.

Asie: tous les pays sauf Israël, le Japon et la Russie, mais y compris les nouveaux Etats d'Asie centrale, Chypre et la Turquie.

Europe: Malte et les pays issus de l'ex-Yougoslavie.

Océanie: tous les pays sauf l'Australie et la Nouvelle-Zélande.

\section{c) Classification économique des pays du Tiers Monde}

\section{Principaux exportateurs de pétrole}

Algérie*, Angola, Arabie Saoudite*, Bahrein, Brunéi Darussalam, Congo, Emirats arabes unis*, Gabon, Indonésie*, Iran*, Irak*, Koweït, Libye*, Nigéria*, Oman, Qatar*, Syrie, Trinité-et-Tobago, Vénézuela* et Yémen.

* Pays membres de l'OPEP

Cette liste de pays a été révisée par rapport à l'année précédente (voir point $d$ ) ci-dessous)

\section{Principaux exportateurs d'articles manufacturés}

Brésil, Chine, Chine RAS de Hong Kong, Corée du Sud, Inde, Malaisie, Mexique, Philippines, Singapour, Taiwan, Thaillande et Turquie.

Cette liste de pays a été révisée par rapport à l'année précédente (voir point $d$ ) ci-dessous)

Autres pays

Tous les pays ou territoires du Tiers Monde non-compris dans les deux groupes précédents. 


\section{d) Remarques}

- La CNUCED révise périodiquement sa classification afin de l'adapter à l'évolution des structures économiques et politiques du monde. Il convient d'en tenir compte lorsqu'on compare les statistiques commerciales actuelles aux chiffres publiés antérieurement.

๖ Une série de changements sont indiqués dans l'Annuaire Suisse-Tiers Monde 1994. Une nouvelle modification signalée dans l'Annuaire 1995 concerne le transfert de la Malaisie, de la Thaïlande et de la Turquie dans le groupe des principaux pays exportateurs d'articles manufacturés. Enfin, l'Annuaire 1997 intègre l'ancien groupe des «pays socialistes d'Asie» dans celui des pays du Tiers-Monde. Les républiques d'Asie et du Caucase de l'ancienne URSS sont désormais aussi comprises dans ce groupe.

- Les changements suivants font que les statistiques pour l'année 1999 de cet annuaire ne correspondent pas aux chiffres publiés dans l'Annuaire de l'année précédente:

- Principaux exportateurs de pétrole: le Yémen a été inclus dans le groupe, alors que l'Equateur a été retiré de cette liste de pays.

- Principaux exportateurs d'articles manufacturés. Les pays de l'ancienne Yougoslavie ne font plus partie de ce groupe, alors que la Chine, l'Inde et les Philippines ont été ajoutés.

- Le Sénégal a été ajouté à la liste des pays les moins avancés - PMA.

\section{B. STATISTIQUES DES FLUX FINANCIERS ET DE L'AIDE PUBLIQUE AU DÉVELOPPEMENT}

Le CAD a établi une liste des pays bénéficiaires de l'aide. Il la révise régulièrement pour tenir compte du fait que les pays sont de plus en plus nombreux à sortir de la catégorie des pays en développement. Le CAD distingue depuis quelques années les pays de la liste I, pays en développement, dont l'aide est comprise dans l'APD-Aide au pays en développement, et les pays de la liste II, pays en transition. L'aide vers ces pays est comprise dans un agrégat statistique séparé « d'aide publique».

La partie I de la liste comprend les pays en développement. La partie II se compose quant à elle des pays en transition, c'est-à-dire des plus avancés parmi les pays de l'Est et les pays en développement.

Les listes complètes des pays en développement et des pays en transition de la partie II se trouve à la fin du présent Annuaire. En voici les grandes lignes:

\section{a) Pays membres du Comité d'aide au développement, CAD}

Allemagne, Australie, Autriche, Belgique, Canada, Danemark, Espagne, EtatsUnis d'Amérique, Finlande, France, Irlande, Italie, Japon, Luxembourg, Norvège, Nouvelle-Zélande, Pays-Bas, Portugal, Royaume-Uni, Suède et Suisse. La Commission des Communautés européennes est également membre du CAD. 


\section{b) Pays en développement (partie I de la liste du CAD)}

Afrique: tous les pays, y compris l'Afrique du Sud.

Amérique: tous les pays sauf le Canada et les Etats-Unis.

Cependant, le CAD a transféré les Bahamas dans le groupe des pays en transi-

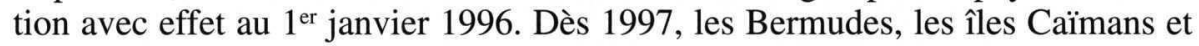
les îles Falkland ont été transférées dans le groupe des pays en transition.

Asie: tous les pays sauf le Japon, la Russie et la Turquie, mais y compris les Etats d'Asie centrale.

Cependant, le CAD a transféré Brunei, les Emirats arabes unis, Koweït, Qatar et Singapour dans le groupe des pays en transition avec effet au $1^{\text {er }}$ janvier 1996 (voir ci-dessous). Dès le $1^{\text {er }}$ janvier 1997, le CAD a aussi transféré Chypre, les Emirats arabes unis, Hongkong, Israël, le Taïpei chinois dans le groupe des pays en transition.

Europe: Albanie, Bosnie-Herzégovine, Croatie, Gibraltar, Malte, Moldavie, Serbie/Monténégro, Macédoine, Slovénie, Grèce, Chypre et Turquie.

Océanie: tous les pays sauf l'Australie et la Nouvelle-Zélande.

c) Pays en transition (partie II de la liste du CAD) (liste applicable aux flux de ressources dès 1996)

Pays de l'Est

Europe: Bélarus, Bulgarie, Estonie, Hongrie, Lettonie, Lituanie, Pologne, Roumanie, Russie, Slovaquie, Tchéquie et Ukraine.

La Moldavie a été transférée dans la liste des pays en développement dès le $1^{\text {er janvier } 1997 .}$

Pays et territoires en développement plus avancés

Amérique: Bahamas, Bermudes, îles Caïmans, îles Falkland.

Asie: Brunei, Chypre, Emirats arabes unis, Hongkong, Israël, Koweït, Qatar, Singapour et Taïpei chinois.

Voir listes complètes à la fin de l'Annuaire.

\section{d) Interprétation des chiffres}

Chaque année, le CAD réalise une enquête auprès des Etats membres concernant les ressources mises à la disposition des pays en développement et des pays en transition. On observe des distorsions importantes:

- les flux de capitaux (à l'exception des remboursements) qui résultent d'opérations financières effectuées par les résidents des pays en développement ou en transition ne sont pas recensés; 
- les apports nets de ressources (appelés aussi versements nets ou encore flux nets) ne correspondent pas à des transferts nets. Les remboursements et les rapatriements de capitaux sont déduits, mais pas les paiements d'intérêts et de dividendes. Les réinvestissements de bénéfices sont comptés comme des apports de ressources.

En outre, les séries chronologiques ne sont souvent pas révisées suite aux modifications apportées à la classification des pays.

\subsection{PRINCIPALES SOURCES}

a) Publications suisses

BNS - Les banques suisses, Zurich (parution annuelle) www.snb.ch BNS - Bulletin mensuel, Zurich rubrique «Publications»

DIRECTION GÉNÉRALE DES DOUANES

Statistique du commerce extérieur de la Suisse. Statistique annuelle, Berne www.zoll.admin.ch, rubrique «Commerce extérieur»

DDC et SECO - Coopération au développement de la Confédération suisse: Rapport annuel, Berne (parution annuelle)

La Vie économique, Revue de politique économique, Berne (parution mensuelle) OFFICE FÉDÉRAL DE LA STATISTIQUE www.statistik.admin.ch

\section{b) Publications d'organisations internationales}

CNUCED - Manuel de statistiques du commerce international et du développement, New York, Nations Unies (parution annuelle)

OCDE - Coopération pour le développement, Efforts et politiques des Membres du Comité d'Aide au Développement, Paris (parution annuelle)

OCDE - Répartition géographique des ressources financières mises à la disposition des pays en développement, Paris (parution annuelle)

ONU - Bulletin mensuel de statistiques, New York, Nations Unies 